\title{
Effects of polishing on surface roughness and hardness of glass-fiber- reinforced polypropylene
}

\author{
Yasuhiro TANIMOTO and Manamu NAGAKURA \\ Department of Dental Biomaterials, Nihon University School of Dentistry at Matsudo, 2-870-1 Sakae-cho Nishi, Matsudo, Chiba 271-8587, Japan \\ Corresponding author, Yasuhiro TANIMOTO; E-mail: tanimoto.yasuhiro@nihon-u.ac.jp
}

\begin{abstract}
Removable partial dentures (RPDs) with resin-clasp retentive parts, which are known as non-metal-clasp dentures (NMCDs), have been used as alternatives for conventional RPDs with metal clasp, in case of aesthetic prosthodontic treatments. In this study, a profilometer and dynamic micro-indentation tests were used to investigate the effects of polishing on the surface properties such as surface roughness (Ra), dynamic hardness, and elastic modulus of high-rigidity glass-fiber-reinforced thermoplastics (GFRTPs) composed of E-glass fibers and polypropylene for NMCDs. The Ra values of the GFRTPs after polishing were significantly lower than those before polishing. The values were close to the Ra threshold level of 0.2 , which corresponds to an acceptable surface smoothness for denture base materials. Polishing did not significantly change the dynamic hardnesses and elastic moduli of the GFRTPs. The fiber loading did not greatly affect the micromechanical properties of the GFRTPs because the glass-fiber reinforcement is embedded in the polypropylene matrix.
\end{abstract}

Keywords: Fiber-reinforced thermoplastics, Non-metal-clasp dentures, Surface roughness, Dynamic micro-indentation method, Dynamic hardness

\section{INTRODUCTION}

Removable partial dentures (RPDs) with resin-clasp retentive parts, which are known as non-metal-clasp dentures (NMCDs), have been used as alternatives for conventional RPDs with metal clasp, in case of aesthetic prosthodontic treatments ${ }^{1}$. In a previous study, highrigidity glass-fiber-reinforced thermoplastics (GFRTPs) composed of E-glass fibers and polypropylene for NMCDs were developed using an injection-molding method $^{2}$. Figure 1 shows a photograph of a conventional RPD with metal frameworks, an NMCD without metal frameworks, and a novel NMCD made from GFRTP. The RPD with metal frameworks is unesthetic because of its metallic color and has the potential to cause allergic reactions. In contrast, a conventional NMCD without metal frameworks is often much more esthetically pleasing than an RPD with metal frameworks and can be used for patients who are allergic to metal. However, the use of an NMCD without metal frameworks can seriously affect the remaining tissues because of its low rigidity, and such an NMCD does not conform to the standard principles of RPD design ${ }^{3)}$. A novel NMCD made from the GFRTPs designed in a previous study is esthetically pleasing and has sufficient rigidity, and its properties can be controlled based on the fiber loading and pigmentation ${ }^{4}$. Furthermore, the GFRTPs have clinically acceptable color stability and might be satisfactory for clinical use ${ }^{5}$.

In addition to evaluation of their esthetic appearance, flexural properties, and color stability, the surface properties of GFRTPs, such as surface roughness and hardness, must be evaluated for clinical use in prosthodontic treatment. The surface roughness not only affects patient comfort and denture discoloration but can also contribute to bacterial adhesion and biofilm formation, and consequently periodontal disease ${ }^{6,7)}$. Some of the reported disadvantages of early forms of NMCDs include a tendency to surface roughening, discoloration, and loss of luster after a short period of time ${ }^{8,9)}$. The hardness also affects the surface properties of denture base materials because it improves prosthesis finishing and maximizes its resistance to abrasion and scratching during service ${ }^{10)}$. Additionally, the elastic modulus, which is related to the stiffness of a material, can affect the wear resistance. The surface of an NMCD can be easily damaged because the surface is not as hard as that of polymethyl methacrylate (PMMA) dentures ${ }^{3)}$. Mechanical polishing can easily change the surface roughness of denture base materials; it is intended to produce wear of the surface, thereby reducing the surface roughness of the materials. However, the effects of mechanical polishing on the surface properties of GFRTPs, such as surface roughness, hardness, and elastic modulus, are unknown.

Micro-indentation hardness testing is a convenient method for investigating the mechanical properties of a small volume of material ${ }^{11,12}$. The dynamic microindentation method has significant advantages over conventional hardness tests. This depth-sensing indentation hardness test can continuously measure the load on, and the displacement of, the indenter until a given maximum load or depth is reached and then removed, without the need for visualization of indentation. The mechanical parameters of a material, such as the dynamic hardness and elastic modulus, can also be obtained by this method.

The purpose of this study was to investigate the effects of polishing on the surface properties of GFRTPs, such as surface roughness, dynamic hardness, and 


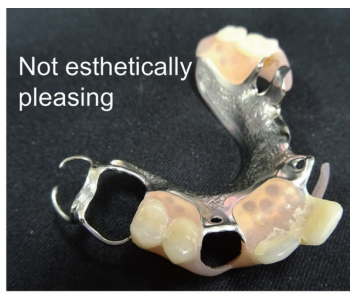

RPD with metal frameworks

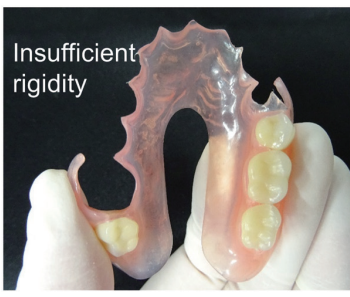

RPD without metal frameworks (Conventional NMCD)

Fig. 1 Photographs of conventional RPD with metal frameworks, RPD without metal frameworks (i.e., NMCD), and novel GFRTP-based NMCD.

modulus, using a profilometer and a dynamic microindentation tester. The first hypothesis was that polishing would affect the surface roughness; the second hypothesis was that polishing would not affect the dynamic hardness and elastic modulus.

\section{MATERIALS AND METHODS}

\section{Preparation of GFRTPs}

GFRTPs composed of E-glass fibers, a polypropylene matrix, and a small amount of coloring pigment were produced by injection-molding pellets (Plastron, PPGF50-02, Daicel Polymer, Tokyo, Japan) consisting of polypropylene reinforced with E-glass fibers of diameter $17 \mu \mathrm{m}$ and length $10 \mathrm{~mm}$, and 2 mass\% of pigment (Aesthetic Intensive-Colors: Purpur Red, Candulor, Glattpark, Switzerland); this process is described in detail elsewhere ${ }^{2,5)}$. According to a previous study on the optimum fiber contents of GFRTPs, GFRTPs were prepared with fiber contents of 0 mass\% (GF0), 10 mass\% (GF10), and 20 mass\% (GF20). Two commercially available NMCD materials, namely Valplast (VA; Unival, Tokyo, Japan) and EstheShot Bright (EB; i-Cast, Kyoto, Japan), and two commercially available PMMA denture base materials, namely Polybase II (PB; Nissin, Kyoto, Japan) and Ivocap (IC; Ivoclar Vivadent, Tokyo, Japan), were used as controls. These specimens were cut to shapes of length $10 \mathrm{~mm}$, width $10 \mathrm{~mm}$, and thickness $3.0 \mathrm{~mm}$. Finally, each specimen was polished with 1000grit $\mathrm{SiC}$ paper under running water.

\section{Surface roughness ( $R$ a) evaluation}

The surface roughness ( $\mathrm{Ra}$ ) values of each sample before and after polishing were determined using a contact profilometer (DR130, Sato Shoji, Kanagawa, Japan).

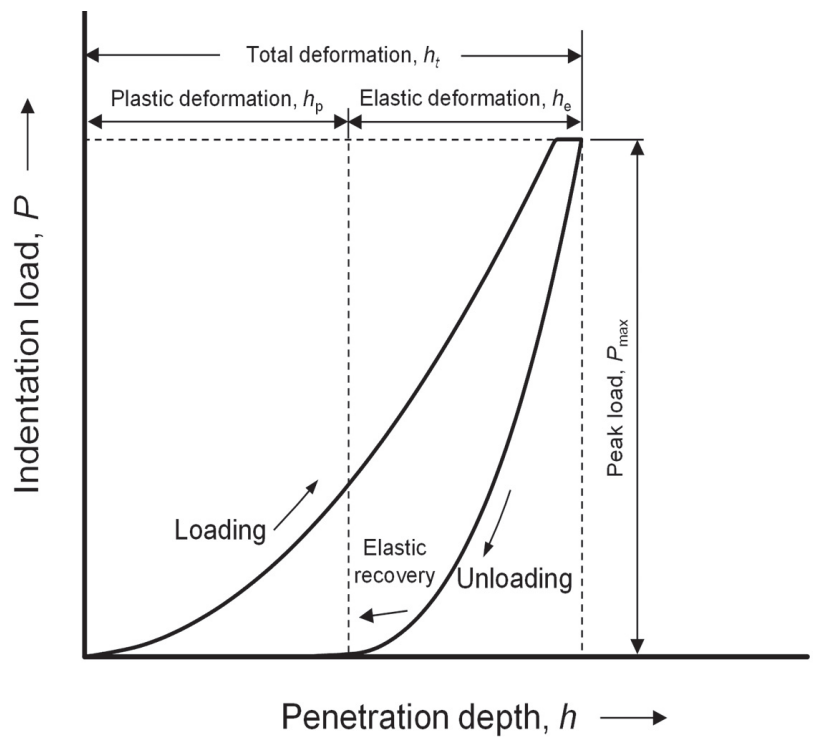

Fig. 2 Micro-indentation load versus penetration depth curve obtained from dynamic ultra-micro-hardness tests.

This instrument measures small surface variations by moving a diamond stylus in contact with the surface laterally across the sample. The vertical displacement of the stylus indicates the surface variations, usually measuring from 0.05 to $10.0 \mu \mathrm{m}$. The height position of the diamond stylus is converted to a digital signal, which is stored and displayed. The profilometer was calibrated with a cutoff length of $0.80 \mathrm{~mm}$ and an evaluation length of $4.0 \mathrm{~mm}$. The Ra values were the average for six specimens $(n=6)$, which were each measured three times.

\section{Dynamic micro-indentation test}

A dynamic ultra-micro-hardness tester (DUH-211, Shimadzu, Kyoto, Japan) fitted with a Berkovich indenter tip was used to investigate the mechanical behavior of the GFRTP surface. The specimens were fixed to an attached holder. Dynamic micro-indentation primarily involves application of a controlled load $(P)$ through a diamond tip that is in contact with a smooth surface. The penetration depth $(h)$ of the indentation is continuously recorded as a function of load. Figure 2 shows a typical micro-indentation load-penetration depth curve obtained from dynamic micro-indentation tests. During indenter loading and unloading, the specimen is subjected to both plastic deformation $\left(h_{\mathrm{p}}\right)$ and elastic deformation $\left(h_{\mathrm{e}}\right)$. The total deformation $\left(h_{\mathrm{t}}\right)$ is the sum of $h_{\mathrm{p}}$ and $h_{\mathrm{e}}$ in the micro-indentation loadpenetration depth curve. The dynamic hardness and elastic modulus can be obtained from the indentation load and penetration depth data ${ }^{11,12)}$.

The dynamic hardness $(\mathrm{DH})$ of the sample was calculated from the following equation:

$\mathrm{DH}=\alpha P / h^{2}$, where $\alpha$ is a geometrical constant of the Berkovich 
indenter (3.8584), $P$ is the applied load during the indentation test, and $h$ is the indentation penetration depth.

The elastic modulus $(E)$ of the sample was calculated from the following equation:

$$
1 / E_{\mathrm{r}}=\left(1-V^{2}\right) / E+\left(1-V_{\mathrm{i}}^{2}\right) / E_{\mathrm{i}},
$$

where $E_{\mathrm{r}}$ is the reduced elastic modulus from the indenter, $V$ is Poisson's ratio for the sample, $V_{\mathrm{i}}$ is Poisson's ratio for the Berkovich indenter (0.07), and $E_{\mathrm{i}}$ is the modulus of the Berkovich indenter (1,140 GPa).

According to a previous study ${ }^{2)}$, dynamic microindentation tests were performed with peak loads $\left(P_{\max }\right)$ of $50.0 \mathrm{mN}$. As well, the load rate was kept constant at $13.3 \mathrm{mN} / \mathrm{s}$ and the hold time at the maximum load was set at $15 \mathrm{~s}$. The dynamic micro-indentation results, i.e., the dynamic hardness and elastic modulus, were obtained as the average values of six specimens $(n=6)$, which were each measured three times.

\section{Statistical analysis}

The experimental results were analyzed using analysis of variance and Scheffé's multiple comparison test among the means at $p=0.05$.

\section{RESULTS}

Figure 3 shows the Ra values of the GFRTPs and controls, before and after polishing. Before polishing, the Ra values of GF0, GF10, GF20, VA, EB, PB, and IC were $1.19 \pm 0.34,1.65 \pm 0.41,1.40 \pm 0.81,0.61 \pm 0.05,0.80 \pm 0.23$, $0.72 \pm 0.25$, and $1.39 \pm 0.18$, respectively. There were significant differences between the Ra values of GF10 and VA or PB before polishing $(p<0.05)$. After polishing,

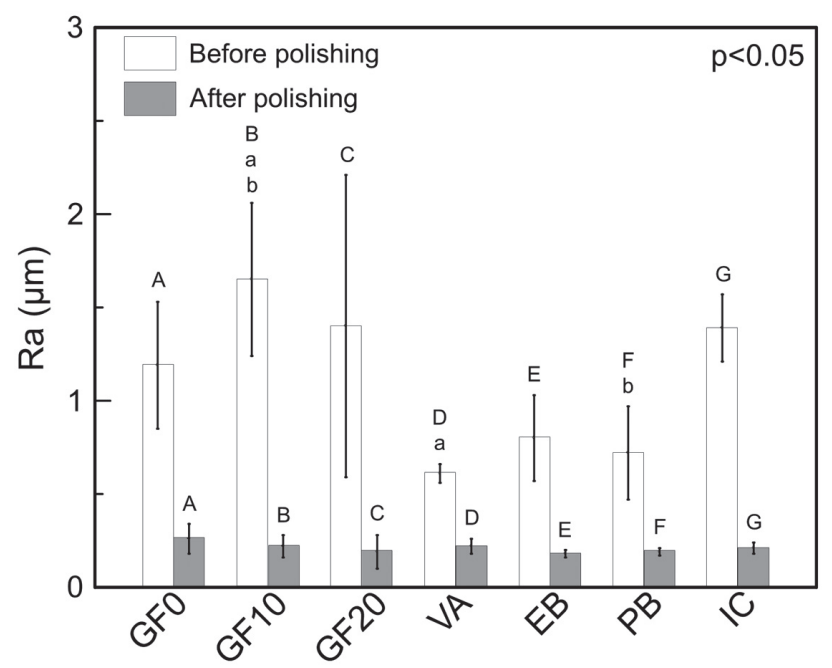

Fig. 3 Surface roughnesses of GFRTPs and controls before and after polishing.

Same lower-case letters indicate significant differences among samples before or after polishing at $p<0.05$. Same upper-case letters indicate significant differences between each sample before and after polishing at $p<0.05$. the Ra values of GF0, GF10, GF20, VA, EB, PB, and IC were $0.26 \pm 0.08,0.22 \pm 0.06,0.19 \pm 0.09,0.22 \pm 0.04$, $0.18 \pm 0.02,0.19 \pm 0.02$, and $0.21 \pm 0.03$, respectively. There were no significant differences among the Ra values of the samples after polishing $(p>0.05)$. For all the samples, there were significant differences between the Ra values

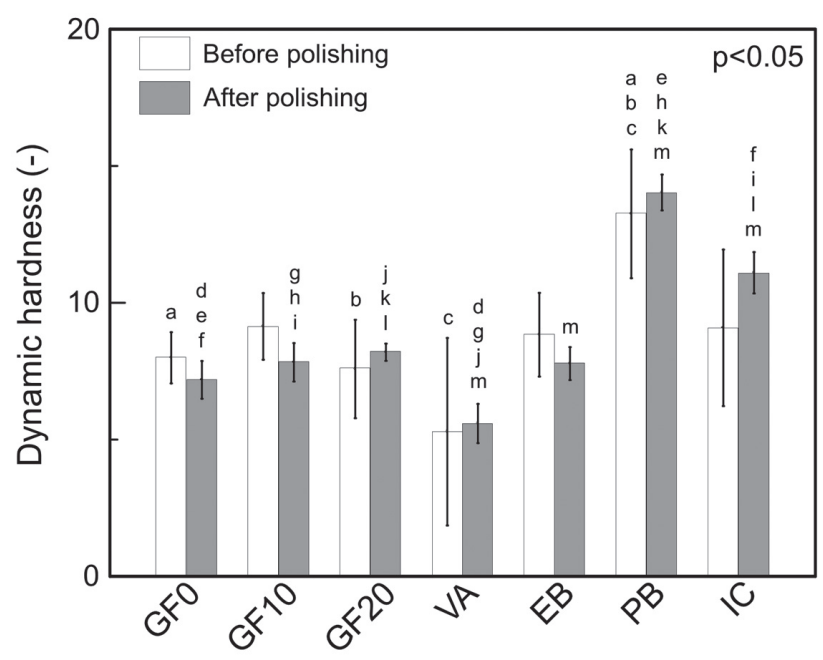

Fig. 4 Dynamic hardnesses of GFRTPs and controls before and after polishing.

Same lower-case letters indicate significant differences among samples before or after polishing at $p<0.05$. Same upper-case letters indicate significant differences between each sample before and after polishing at $p<0.05$.

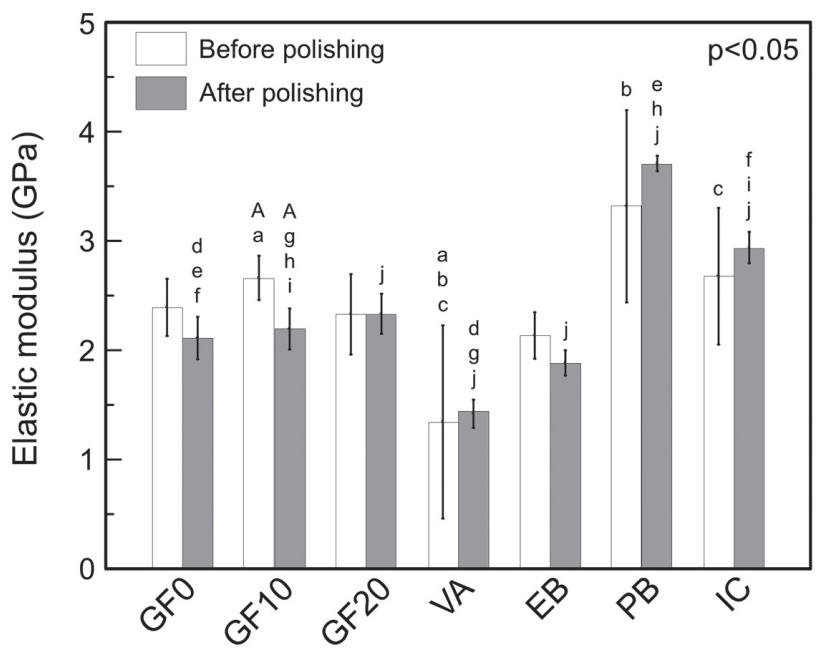

Fig. 5 Elastic moduli of GFRTPs and controls before and after polishing.

Same lower-case letters indicate significant differences among samples before or after polishing at $p<0.05$. Same upper-case letters indicate significant differences between each sample before and after polishing at $p<0.05$. 

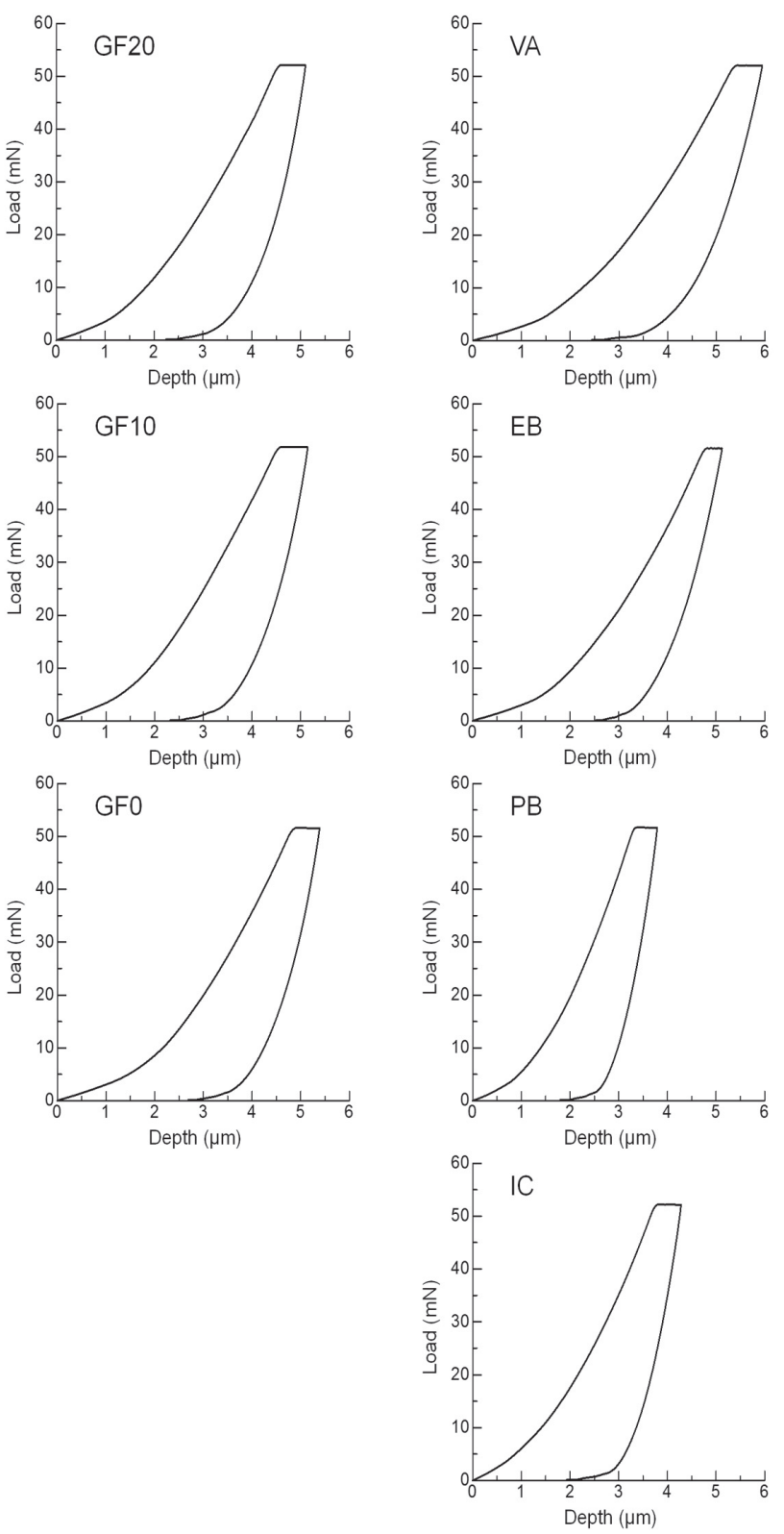

Fig. 6 Typical indentation load-penetration depth curves of GFRTPs and controls after polishing, obtained from dynamic ultra-micro-hardness tests.

before and after polishing $(p<0.05)$.

Figure 4 shows the dynamic hardnesses of the GFRTPs and controls before and after polishing. Before polishing, the dynamic hardnesses of GF0, GF10, GF20, VA, EB, PB, and IC were 7.99 $\pm 0.94,9.14 \pm 1.22,7.58 \pm 1.80$, $5.29 \pm 3.43, \quad 8.83 \pm 1.53, \quad 13.25 \pm 2.35$, and $9.08 \pm 2.86$, respectively. After polishing, the dynamic hardnesses of GF0, GF10, GF20, VA, EB, PB, and IC were $7.18 \pm 0.69$, $7.83 \pm 0.70,8.19 \pm 0.32,5.59 \pm 0.72,7.78 \pm 0.60,14.03 \pm 0.66$, and $11.10 \pm 0.76$, respectively. The dynamic hardness of PB was highest, and that of VA was lowest. For all samples, there was no significant difference between

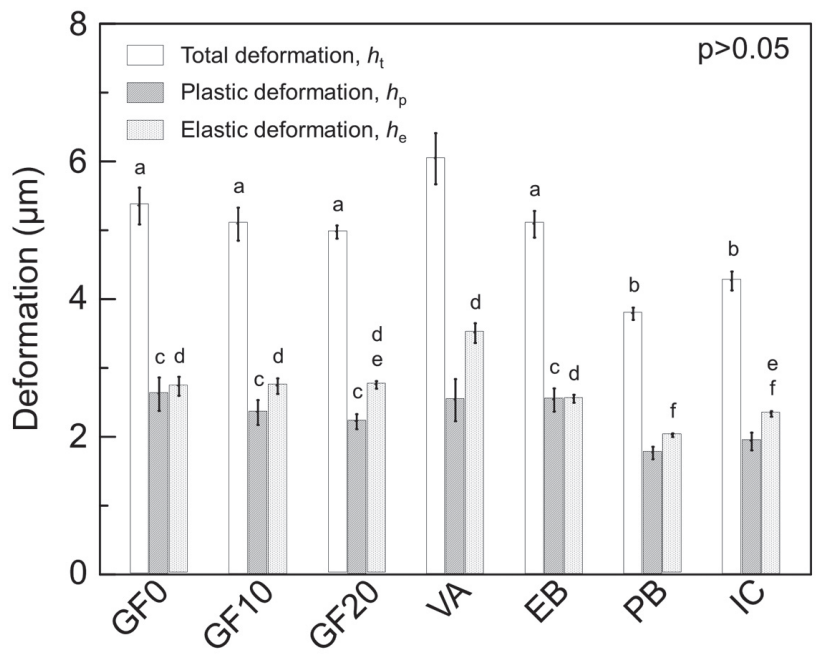

Fig. 7 Total, plastic, and elastic deformations of GFRTPs and controls after polishing.

Same lower-case letters indicate no significant differences among samples at $p>0.05$.

the dynamic hardnesses before and after polishing $(p>0.05)$.

Figure 5 shows the elastic moduli of the GFRTPs and controls before and after polishing. Before polishing, the elastic moduli of GF0, GF10, GF20, VA, EB, PB, and IC were $2.39 \pm 0.26,2.66 \pm 0.20,2.33 \pm 0.37,1.34 \pm 0.88$, $2.13 \pm 0.21,3.32 \pm 0.88$, and $2.68 \pm 0.63$, respectively. After polishing, the elastic moduli of GF0, GF10, GF20, VA, $\mathrm{EB}, \mathrm{PB}$, and IC were $2.11 \pm 0.19,2.19 \pm 0.19,2.33 \pm 0.18$, $1.42 \pm 0.13, \quad 1.88 \pm 0.12, \quad 3.71 \pm 0.07, \quad$ and $2.94 \pm 0.14$, respectively. As in the case of dynamic hardness, the elastic modulus of $\mathrm{PB}$ was highest, and that of VA was lowest. Additionally, there were no significant differences between the elastic modulus of each sample before and after polishing, except for GF10 ( $p>0.05)$.

Figure 6 shows typical indentation load-penetration depth curves for the GFRTPs and controls after polishing. The load-penetration depth curves indicate that penetration of the GFRTPs tended to decrease with increasing fiber content. The indentation loadpenetration depth curves for all the samples indicate specimen creep at a peak load of $50.0 \mathrm{mN}$ for $15 \mathrm{~s}$.

Figure 7 shows the $h_{\mathrm{t}}$ values, which are the sum of $h_{\mathrm{p}}$ and $h_{\mathrm{e}}$, of the GFRTPs and controls after polishing. Here, indentation deformation, $h_{\mathrm{t}}=h_{\mathrm{p}}+h_{\mathrm{e}}$, is always satisfied. VA showed the largest $h_{\mathrm{t}}$ value among the samples $(p<0.05)$. The ratios of elastic deformation to total deformation (i.e., $h_{\mathrm{e}} / h_{\mathrm{t}}$ ) for GF0, GF10, GF20, VA, $\mathrm{EB}, \mathrm{PB}$, and IC were 51.1, 53.8, 55.4, 58.2, 50.2, 53.4, and 54.7 , respectively. VA also had the highest $h_{\mathrm{e}} / h_{\mathrm{t}}$ ratio among the samples, i.e., VA showed the highest elastic recovery of the samples under unloading.

\section{DISCUSSION}

It is important that the surface properties of denture 
base materials are determined before their use in the mouth because surface roughening can cause discoloration of, and biofilm formation on, the denture, and this can cause discomfort to patients. In the present study, the surface properties before and after polishing of GFRTPs for use in NMCDs, such as surface roughness, dynamic hardness, and elastic modulus, were investigated.

First, the surface roughnesses of the GFRTPs before and after polishing were evaluated by using profilometry to determine their Ra values. Contact profilometers have been used to obtain Ra values in previous studies ${ }^{13,14}$. The Ra values of all the samples were significantly lower after polishing than those before polishing (Fig. 3), i.e., polishing clearly decreased the Ra values of all the samples. The first null hypothesis was confirmed, because polishing affected the surface roughness. Additionally, there were no significant differences among the Ra values of the samples after polishing. These results show that GFRTPs with smooth surfaces similar to those of PMMA can be obtained. Bollen et al. ${ }^{15)}$ suggested that $\mathrm{Ra}=0.2$ is an acceptable threshold level for the surface smoothness of denture base materials, because further reduction in plaque accumulation is not achieved by more extensive polishing. In the present study, the experimental $\mathrm{Ra}$ values for the GFRTPs with fiber contents of 10 and 20 mass\%, i.e., 0.19 and 0.22 , respectively, were close to the acceptable $\mathrm{Ra}$ value, i.e., 0.2. This indicates that GFRTPs with clinically acceptable surface roughnesses can be obtained by polishing.

Secondly, the dynamic hardnesses and elastic moduli of the GFRTPs were calculated from indentation load-penetration depth data. Depth-sensing microindentation testing is a useful method for investigating the mechanical properties of dental materials on a small scale ${ }^{16-18)}$. In particular, this method can be used to measure the degree of elastic behavior, in addition to the dynamic hardness and elastic modulus of a material. The dynamic hardnesses and elastic moduli of the GFRTPs were lower than those of PMMA denture base materials ( $\mathrm{PB}$ and $\mathrm{IC}$ ) but equal to or higher than those of conventional NMCD materials (VA and EB) (Figs. 4 and 5). However, polishing did not significantly change the dynamic hardnesses and elastic moduli of all the samples. The second null hypothesis was also confirmed, because polishing did not generally affect the dynamic hardness and elastic modulus. It has been reported that below a certain threshold $\mathrm{Ra}$, the elastic moduli are similar and not greatly affected by surface roughness ${ }^{19)}$. It is therefore assumed that surface roughnesses below those obtained in the present study do not affect the micromechanical properties such as dynamic hardness and elastic modulus. There were no significant differences among the dynamic hardnesses or elastic moduli of GFRTPs with different fiber contents, although the fiber content did signifcantly affect the mechanical properties such as flexural strength and modulus, which were obtained by the flexural test in a previous study ${ }^{2}$. It is considered that the fiber content did not affect the micromechanical properties of the GFRTPs with fiber contents $<20$ mass $\%$ because the GFRTP surface is polypropylene rich. This finding regarding the effect of fiber content on surface properties agrees with the results of our previous study ${ }^{2}$.

Generally, NMCD materials for use in clasp construction must have sufficiently high elasticity and rigidity ${ }^{20)}$. The retentive clasp arms must have elasticity and should not be permanently deformed during insertion or removal of the denture and should have sufficient rigidity to retain the denture satisfactorily. It is therefore important for prosthodontists to have reliable information on the mechanical properties of NMCDs, e.g., their elastic and plastic behaviors. The dynamic micro-indentation method can continuously record the penetration depth of a specimen during dynamic loading and unloading at the indentation tip and provide information on the degrees of elastic and plastic deformation (Figs. 6 and 7). The $h_{\mathrm{e}} / h_{\mathrm{t}}$ ratios of the GFRTPs tended to increase with increasing fiber content; GF20 had the highest $h_{\mathrm{e}} / h_{\mathrm{t}}$ ratio, indicating a higher degree of elastic behavior compared with the other GFRTPs. Hirayama et al. ${ }^{11}$ evaluated the micromechanical properties of flowable resin composites by the dynamic micro-indentation method. The authors reported that a composite based on urethane dimethacrylate had a higher degree of plastic behavior compared with a composite based on bisphenol-Aglycidyl methacrylate. Nagai et al. ${ }^{12)}$ investigated the micromechanical behaviors of orthodontic wire alloys by the dynamic micro-indentation method. The authors reported that high elastic recovery associated with superelastic behavior on the surface of a nickel-titanium alloy was detected by indentation load-penetration depth curves obtained using a dynamic ultra-micro-hardness tester. The results obtained in the present study indicate that a dynamic ultra-micro-hardness tester could detect differences among the elastic recoveries of the surfaces of GFRTPs with various fiber contents. However, more detailed analysis of the elastic, plastic, and creep behaviors of GFRTPs is needed.

Finally, the surfaces of NMCD materials are more easily damaged than those of PMMA; however, these NMCD materials are regarded as being not susceptible to surface loss, even when damaged ${ }^{3)}$. This means that NMCD materials may be more difficult to polish than PMMA $^{13)}$. Although it was indicated that GFRTPs with clinically acceptable surface roughnesses can be obtained by polishing, further investigations are therefore needed to establish polishing techniques that are suitable for GFRTPs for clinical use in metal-free RPDs.

\section{CONCLUSIONS}

This study investigated the surface properties before and after polishing of GFRTPs for use in NMCDs, by evaluating their surface roughnesses, dynamic hardnesses, and elastic moduli. The results and conclusions are summarized as follows.

1. The surface roughnesses (Ra) of the GFRTPs were assessed by profilometry. The Ra values of 
the GFRTPs after polishing were significantly lower than those before polishing. The Ra values of the GFRTPs after polishing were close to the Ra threshold level that is clinically acceptable for the surface smoothness of denture base materials.

2. The dynamic hardnesses and elastic moduli of the GFRTPs were evaluated by microindentation testing. Polishing did not affect the micromechanical properties such as dynamic hardness and elastic modulus. The fiber loading did not affect the micromechanical properties of the GFRTPs because the glass-fiber reinforcement was embedded in the polypropylene matrix.

\section{ACKNOWLEDGMENT}

This work was supported by JSPS KAKENHI Grant Number JP15K11202.

\section{REFERENCES}

1) Fueki K, Yoshida-Kohno E, Wakabayashi N. Oral healthrelated quality of life in patients with non-metal clasp dentures: a randomised cross-over trial. J Oral Rehabil 2017; 44: 405-413.

2) Nagakura M, Tanimoto Y, Nishiyama Y. Fabrication and physical properties of glass-fiber-reinforced thermoplastics for non-metal-clasp dentures. J Biomed Mater Res B 2017; 105: 2254-2260.

3) Fueki K, Ohkubo C, Yatabe M, Arakawa I, Arita M, Ino S, Kanamori T, Kawai Y, Kawara M, Komiyama O, Suzuki T, Nagata K, Hosoki M, Masumi S, Yamauchi M, Aita H, Ono T, Kondo H, Tamaki K, Matsuka Y, Tsukasaki H, Fujisawa M, Baba K, Koyano K, Yatani H. Clinical application of removable partial dentures using thermoplastic resin. Part I: Definition and indication of non-metal clasp dentures. J Prosthodont Res 2014; 58: 3-10.

4) Tanimoto $Y$, Nagakura M, Nishiyama N. Glass fiberreinforced thermoplastics for use in metal-free removable partial dentures: Combined effects of fiber loading and pigmentation on color differences and flexural properties. J Prosthodont Res 2018; 62: 359-364.

5) Nagakura M, Tanimoto Y, Nishiyama N. Color stability of glass-fiber-reinforced polypropylene for non-metal clasp dentures. J Prosthodont Res 2018; 62: 31-34.

6) Gad MM, Fouda SM, ArRejaie AS, Al-Thobity AM. Comparative effect of different polymerization techniques on the flexural and surface properties of acrylic denture base. J Prosthodont 2017; doi:10.1111/jopr.12605.
7) Heimer S, Schmidlin PR, Stawarczyk B. Discoloration of PMMA, composite, and PEEK. Clin Oral Investig 2017; 21: 1191-1200.

8) Kawara M, Iwata Y, Iwasaki M, Komoda Y, Iida T, Asano T, Komiyama O. Scratch test of thermoplastic denture base resins for non-metal clasp dentures. J Prosthodont Res 2014; 58: $35-40$

9) Sepulveda-Navarro WF, Arana-Correa BE, Borges CP, Jorge JH, Urban VM, Campanha NH. Color stability of resins and nylon as denture base material in beverages. J Prosthodont 2011; 20: 632-638.

10) Moussa AR, Dehis WM, Elboraey AN, ElGabry HS. A comparative clinical study of the effect of denture cleansing on the surface roughness and hardness of two denture base materials. Maced J Med Sci 2016; 4: 476-481.

11) Hirayama $S$, Iwai $H$, Tanimoto Y.Mechanical evaluation of fiver flowable resin consposites by the dynamic micro-indentation method. J Dent Biomech 2014; 5: 1758736014533983.

12) Nagai $M$, Tanimoto $Y$, Inami $T$, Yamaguchi $M$, Nishiyama $\mathrm{N}$, Kasai K. Effects of indentation load on the mechanical behavior of orthodontic wire alloys by dynamic microindentation method. Int J Oral Med Sci 2013; 12: 41-48.

13) Abuzar MA, Bellur S, Duong N, Kim BB, Lu P, Palfreyman N, Surendran D, Tran VT. Evaluating surface roughness of a polyamide denture base material in comparison with poly (methyl methacrylate). J Oral Sci 2010; 52: 577-581.

14) Berger JC, Driscoll CF, Romberg E, Luo Q, Thompson G. Surface roughness of denture base acrylic resins after processing and after polishing. J Prosthodont 2006; 15: 180186.

15) Bollen CM, Lambrechts P, Quirymen M. Comparison of surface roughness of oral hard materials to the threshold surface roughness for bacterial plaque retention: A review of the literature. Dent Mater 1997; 13: 258-269.

16) Labonte D, Lenz AK Oyen ML. On the relationship between indentation hardness and modulus, ant the damage resistance of biological materials. Acta Biomater 2017; 57: 373-383.

17) Basavarajappa S, Al-Kheraif AA, ElSharawy M, Vallittu PK. Effect of solvent/disinfectant ethanol on the microsurface structure and properties of multiphase denture base polymers. J Mech Behav Biomed Mater 2016; 54: 1-7.

18) Chung SM, Yap AU. Effects of surface finish on indentation modulus and hardness of dental composite restoratives. Dent Mater 2005; 21: 1008-1016.

19) Flury S, Diebold E, Peutzfeldt A, Lussi A. Effect of artificial toothbrushing and water storage on the surface roughness and micromechanical properties of tooth-colored CAD-CAM materials. J Prosthet Dent 2017; 117: 767-774.

20) Tannnous F, Steiner M, Shahin R, Kern M. Retentive forces and fatigue resistance of thermoplastic resin clasps. Dent Mater 2012; 28: 273-278. 